\title{
Mobile Apps and Memes in the Era of COVID-19 Pandemic; Could this Make Quarantine More Acceptable?
}

\author{
Ahmed M Abbas ${ }^{1,2}$, Ebrahim Yousof ${ }^{2,3}$, Mahmoud Kamel ${ }^{2,3}$, Shimaa Selim ${ }^{2,3}$, Safaa Ahmed ${ }^{2,3}$ and \\ Yasmin Ismail ${ }^{2,3}$
}

${ }^{1}$ Department of Obstetrics \& Gynecology, Faculty of Medicine, Assiut University, Egypt

${ }^{2}$ COvid-19 Research of Assiut University Association (CORAUNA) group, Egypt

${ }^{3}$ House-officer, Faculty of Medicine, Assiut University, Egypt

*Corresponding author: Ahmed M Abbas, Department of Obstetrics and Gynecology, Assiut University, Women Health Hospital, 71511, Assiut, Egypt.

To Cite This Article: Ahmed MA, Ebrahim Y, Mahmoud K, Shimaa S, Safaa A, Yasmin I. Mobile Apps and Memes in the Era of COVID-19 Pandemic; Could this Make Quarantine More Acceptable?. 2020 - 9(4). AJBSR.MS.ID.001409. DOI: 10.34297/AJBSR.2020.09.001409.

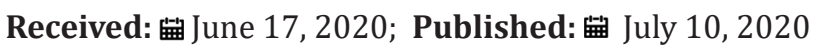

\section{Short Communication}

Coronavirus disease 2019 (COVID-19) pandemic forces the entire world countries to impose a curfew and closes most of the social organizations; that creates a lot of leisure time and the increasing need to fill this space inside the home. Additionally, it increases the need for achieving daily home tasks. Therefore, mobile apps find their way to control most of the people's life from food delivery up to educational lectures and business meetings. That phenomenon provides a lot of benefits; It not only encourages fighting infection but also helps to reduce economic affection from curfew and continuation of working flow. Another significant advantage is that after the end of this pandemic if we replace the traditional way that causes a lot of pollution with these apps, it will be good for the environment, which earns a lot these days from curfew that clearly decreased level of the pollution.

That phenomenon also has its horrible drawbacks; as skilled threat actors are exploiting peoples' concerns about coronavirus to spread mobile malware, including Mobile Remote Access Trojans (MRATs), Banker Trojans, and Premium Dialers, via apps which claim to offer Coronavirus-related information and help for users. MRAT is a type of mobile malware that allows threat actors a complete control and monitoring of a mobile device on which it installed on. Usually, a MRAT is installed on a device to steal data or for surveillance efforts [1].

During the curfew, stress became a daily battle, so managing it is a skill that everyone needs to develop as stress takes a toll on our productivity and, more importantly, our health. We need something that calms people during staying at home to avoid over-thinking, which may lead to fear, stress, and anxiety. Fortunately, new mobile apps can bring stress management easily like Breath2 relax, Pacifica, Happify, Headspace, and Calm.

"Calm" is a mindfulness meditation mobile app that offers a range of mindfulness meditation practice guide modules that vary in length, instruction, and content. The app includes meditation lessons, sleep stories (bed-time stories for grown-ups), sleep music, and nature sounds. "Headspace" A gym membership for the mind," Headspace provides a series of guided meditation sessions and mindfulness training. A free trial is available with additional courses available by subscription. "Happify" A brain-training app based on research showing that some types of activity can help you combat negativity, anxiety, and stress while fostering positive traits like gratitude and empathy [2].

Advantageously, some apps can help by turning our fight against anxiety into a game. They are not really "game" but more like "check here if you did these tasks today." Most of these apps consolidate our health, mood \& energy by small activities, and some give the ability to add friends who you can chat with them and share with them feelings and fears to decrease stress and anxiety. Another group of apps helps people cope with negative things and give them more control over their thoughts and feelings. These all benefits and more exist in apps like "Super Better app" and "Mind Ease." Also, there are apps that can help if anyone have trouble in this second like "quick-relief". 
Apps that help us to face our fears, write down our thoughts to ease our mind, goal settings, health habits, and belief experiment teaches you to experiment to test beliefs that trigger anxiety; all these services and other useful services are in one app like "mind shift." More and more helpful apps like Sanvello, Antistress, and Youper could use during quarantine and curfew [3].

Moreover, there are a lot of apps like "Telemedicine Teladoc," "Primary Care," "Doctor on Demand," "Prescription Management" and "Medisafe Medication Management" serves old aged people and patients to facilitate their time, care about their health and introduce medical bits of advice and prescriptions from home during quarantine [4].

We think that mobile apps can play an important role in make people stay at home easier and useful and help them to overcome their fears, anxiety, and stress. But we should be careful when choosing any app to avoid using malicious or hacker apps. As there is recent research indicates that of the 293 apps claiming to offer treatment for depression and/or anxiety, only $3.41 \%$ had published research on their effectiveness.

Nowadays with the presence of social media platform and the huge number of people using these platforms; about 3.8 billion users and the average person spends about $2 \mathrm{~h}$ and half, according to datareportal.com, only in the US More than $65 \%$ of adults use at least one social media platform [5]. Social media platforms have facilitated information generation, increasing the popularity and spread of memes among online users.

Memes have been so popular in the last few years. Millions of memes are created daily and have been a good way of entertainment [6]. Usually, memes follow the trend sometimes make it. A meme could be a sarcastic commentary on an awkward situation; it could describe a funny situation that goes wrong; it can be educational, can go dark, and goes viral. The current situation of coronavirus disease-19 (COVID-19) crisis and the global quarantine offer good media for both meme makers and the audience.

We owe memes in the current crisis. Memes have helped us in the quarantine passing time, overcome sad moments with a homer, and overcome anxiety. A recent questionnaire done in India tells us that $60 \%$ of participates agreed that looking at humorous messages and memes helps to calm down their anxiety [7].

Memes don't just relieve anxiety in a global crisis, but it also helps to spread knowledge. Rapid exploration of the current memes about COVID-19 found a good amount of information about the virus, how to kill it, hygiene, and more. The problem is one can't rely on this information; however, people depend on social media as a source of information anyway [8]. Health-related information found on the internet affects patient decision-making. Patients also have replaced doctors' advice with searching the web, and we can't ignore the impact of social networks on people's health [8].

Memes have been used in many fields, politics, food, cartoon, everyday life, health, diseases, and more. Memes were even used by social movements to express themselves and to criticizing the opponent's behavior. The use of humor and inside jokes (in memes) enabled, for example, a group of protestors and made them more persuasive [9]. Therefore, why we don't just join the race and share knowledge and empower health practice through memes? We should be open to modern ways of health education. If you are at pandemic, you need something spread faster than a pandemic, a viral meme.

We are lucky that people tend to interact more with memes engaging rational thinking, also when memes touch their emotions. Additionally, it is not a very hard battle to play; however, it is a great tool with great potential. We recommend that trusted health organizations start fighting ignorance with memes now to avoid fighting ignorant health-related memes then.

\section{Conflict of Interest}

The authors state that there are no conflicts of interest.

\section{Reference}

1. Aviran Hazum, Ohad Mana, Israel Wernik, Bogdan Melnykov, Chana Efrati (2020) COVID-19 goes mobile: Coronavirus malicious applications discovered.

2. Rachael Bailey Smith (2020) Effects of Using Mobile App on Perceived Stress During COVID-19 Pandemic.

3. Jake Peterson (2020) Apps That'll Help You Manage Stress \& Anxiety During the Coronavirus Pandemic.

4. Randy Eckhoff, Robert Furberg (2020) Using Mobile Apps to Reduce Stress and Promote Psychological Resilience During COVID-19.

5. Smith A, Anderson M (2018) Social media use in 2018. Pew Research Center: Internet and technology.

6. Coscia M (2014) Average is Boring: How similarity kills a Meme's Success. Sci Rep.

7. Vijayaraghavan P, Singhal D (2020) A Descriptive Study of Indian General Public's Psychological responses during COVID-19 Pandemic Lockdown Period in India.

8. Salathé M, Vu DQ, Khandelwal S, Hunter DR (2013) The dynamics of health behavior sentiments on a large online social network. EPJ Data Science.

9. Underwood P, Welser HT (2011) 'The internet is here' emergent coordination and innovation of protest forms in digital culture. Proceedings of the 2011 iConference pp. 304-311. 